\title{
Hippocampus-Mediated Activation of Superficial and Deep Layer Neurons in the Medial Entorhinal Cortex of the Isolated Guinea Pig Brain
}

\author{
Vadym Gnatkovsky and Marco de Curtis \\ Unit of Clinical Epileptology and Experimental Neurophysiology, Istituto Nazionale Neurologico, 20133 Milan, Italy
}

The entorhinal cortex (EC) is regarded as the structure that regulates information flow to and from the hippocampus. It is commonly assumed that superficial and deep EC neurons project to and receive from the hippocampal formation, respectively. Anatomical evidences suggest that both the hippocampal output and deep EC neurons also project to superficial EC layers. To functionally characterize the interlaminar synaptic EC circuit entrained the by hippocampal output, we performed simultaneous intracellular recordings and laminar profile analysis in the medial EC (m-EC) of the in vitro isolated guinea pig brain after polysynaptic hippocampal activation by lateral olfactory tract (LOT) stimulation. Optical imaging of voltage-generated signals confirmed that the LOT-evoked hippocampusmediated response is restricted to the m-EC. The hippocampal output generated an extracellular current sink in layers V-VI, coupled with an EPSP in deep neurons. Deep neuron firing was terminated by a biphasic IPSP. The earliest response observed in superficial layer neurons was characterized by a feedforward IPSP of circa $100 \mathrm{~ms}(-69 \pm 1.3 \mathrm{mV}$ reversal potential) abolished by local application of $1 \mathrm{~mm}$ bicuculline. The feedforward IPSP was followed by a delayed EPSP blocked by AP-5 (100 $\mu \mathrm{M})$, presumably mediated by deep-tosuperficial m-EC connections.

Our findings demonstrate that superficial m-EC cells are inhibited by the hippocampal output via a feedforward pathway that prevents activity reverberation in the hippocampal-EC-hippocampal loop. We propose that such inhibition could serve as a protective mechanism to prevent epileptic hyperexcitability.

Key words: cortex; learning and memory; electrical stimulation; inhibition; hippocampus; entorhinal

\section{Introduction}

The entorhinal cortex (EC) holds a critical position within the temporal lobe as a gate structure for the bidirectional information flow between the neocortex and hippocampus, which supports memory and spatial orientation functions (Witter, 1993; Squire and Zola, 1996; Burwell, 2000; Witter and Wouterlood, 2002). To understand how the EC and the hippocampus perform such complex operations, it is crucial to analyze and establish the functional interactions between these two structures. The present study will focus on the EC circuit determined by hippocampal activation.

The morphological features of EC neurons and the connection between the EC and hippocampus have been extensively analyzed by anatomical studies (Witter et al., 1989; Insausti et al., 1997; Burwell, 2000, 2001; Witter and Wouterlood, 2002). Mem-

Received Aug. 2, 2005; revised Nov. 24, 2005; accepted Nov. 24, 2005.

This work was partially funded by the Italian Health Ministry (Ricerca Corrente 703901-L1) and by Italian Ministry of University and Research (Fondo per gli Investimenti della Ricerca di Base RBAU01JRCF-001). Multichannel silicon probes were kindly provided by Jemille Hetke from the Center for Neural Communication Technology of the University of Michigan (sponsored by National Institutes of Health National Center for Research Resources Grant P41-RR09754).

Corresponence should be addressed to Marco de Curtis, Unit of Clinical Epileptology and Experimental Neurophysiology, Istituto Nazionale Neurologico, via Celoria 11, 20133 Milano, Italy. E-mail: decurtis@istituto-besta.it. DOI:10.1523/JNEUROSCI.4365-05.2006

Copyright $\odot 2006$ Society for Neuroscience $\quad$ 0270-6474/06/260873-09\$15.00/0 brane and synaptic properties of EC neurons have also been examined in detail on in vitro slice preparations (Jones and Heinemann, 1988; Klink and Alonso, 1993; Jones, 1994; Berretta and Jones, 1996; Dickson et al., 1997; van der Linden and Lopes da Silva, 1998; Hamam et al., 2000; Gloveli et al., 2001; Wang and Lambert, 2003). With reference to the hippocampal projection to the EC, deep neurons represent the main target (Swanson and Cowan, 1977; van Groen et al., 1986), although a fiber contingent has been demonstrated to project directly into superficial layers (Witter, 1993; Tamamaki and Nojyo, 1995; van Haeften et al., 1995).

To date, the reciprocal interactions between the hippocampus and the EC have been studied in vivo by analyzing the responses to local stimulations either within the EC or in the CA1-subiculum (Deadwyler et al., 1975; Finch et al., 1986, 1988; Jones and Heinemann, 1988; Bartesaghi et al., 1989; Lopes da Silva et al., 1990; Isokawa and Finch, 1991; Gloveli et al., 1997b; Kloosterman et al., 2000, 2003b). Similarly, intra-EC deep-to-superficial associative projections (Kohler, 1986; Dolorfo and Amaral, 1998; van Haeften et al., 2003) were analyzed in vivo (Bartesaghi et al., 1989) and in slices (Yang et al., 2004) by analyzing field responses to direct local EC stimulation. The activation of fibers passing in the hippocampal/EC transition region and the direct activation of intrinsic EC fibers induced by these procedures could lead to nonphysiological phenomena, such as antidromic firing, which hinder the correct evaluation of the EC network interactions 
evoked by the hippocampal output. To avoid this problem, we recently developed a new protocol that consists in the repetitive stimulation of the lateral olfactory tract (LOT) in the in vitro isolated guinea pig brain (Biella and de Curtis, 2000). This procedure induces an early activation of the lateral EC that is followed by the invasion of the hippocampus (Biella et al., 2003; Gnatkovsky et al., 2004; Uva and de Curtis, 2005). In these studies, a delayed activation of the EC mediated by the output of the hippocampus was consistently observed during low-rate repetitive LOT stimulation. Interestingly, experiments performed with either optical imaging (Biella et al., 2003) or multielectrode techniques (Gnatkovsky et al., 2004), demonstrated that the delayed hippocampusmediated response was restricted to the caudal and medial part of the EC and was not observed in the lateral/rostral EC. In the present study, repetitive LOT stimulation was used to study the medial EC network activated by the hippocampal output in the isolated guinea pig brain preparation, by performing optical imaging experiments and simultaneous intracellular and laminar profile recordings performed with multichannel silicon probes. The relevance of the findings for the EC-hippocampal loop circuit are discussed.

Preliminary data have been reported previously in abstract form (Gnatkovsky and de Curtis, 2004).

\section{Materials and Methods}

After barbiturate anesthesia $(80 \mathrm{mg} / \mathrm{kg}$ sodium thiopental, i.p.), brains of adult Hartley guinea pigs (150-250 g weight; Charles River, Comerio, Italy) were isolated and transferred to an incubation chamber, according to the previously described technique (Llinás et al., 1981; de Curtis et al., 1991, 1998; Muhlethaler et al., 1993). A saline solution composed of the fol-

lowing (in mM): $126 \mathrm{NaCl}, 3 \mathrm{KCl}, 1.2 \mathrm{KH}_{2} \mathrm{PO}_{4}, 1.3 \mathrm{MgSO}_{4}, 2.4 \mathrm{CaCl}_{2}, 26$ $\mathrm{NaHCO}_{3}, 15$ glucose, and $3 \%$ dextran, molecular weight 70.000 , oxygenated with a $95 \% \mathrm{O}_{2} / 5 \% \mathrm{CO}_{2}$ gas mixture, $\mathrm{pH} 7.3$, was arterially perfused in vitro at $7 \mathrm{ml} / \mathrm{min}$. The experiments were performed at $32^{\circ} \mathrm{C}$.

Extracellular potentials were recorded in the EC with either single glass pipettes filled with $1 \mathrm{M} \mathrm{NaCl}$, or with multichannel silicon probes inserted perpendicular to the cortical lamination (16-channel arranged on a single shaft, separated by $100 \mu \mathrm{m}$; kindly provided by the Center of Neural Communication and Technology of the Michigan University, Ann Arbor, MI). A twisted silver wire was positioned on the LOT to deliver stimuli $(10-25 \mu \mathrm{A} ; 300 \mu \mathrm{s})$ at $0.2-2 \mathrm{~Hz}$. Intracellular recordings were performed with sharp glass microelectrodes filled with $2 \mathrm{M}$ potassium acetate and $1.5 \%$ biocytin (input resistance, $70-100 \mathrm{M} \Omega$ ). In some experiments, glass pipettes were filled with potassium chloride $(\mathrm{KCl} ; 3 \mathrm{M})$ to study IPSP reversal. The position of the recording electrodes was visually controlled with a stereoscopic microscope. Signals were amplified with either a multichannel differential extracellular amplifier (Biomedical Engineering, Thornwood, NY) or with a Neuro Data intracellular amplifier (Neuro Data Instruments, New York, NY). Amplified signals were digitized with an ATMIO-64E3 data acquisition board (National Instru-
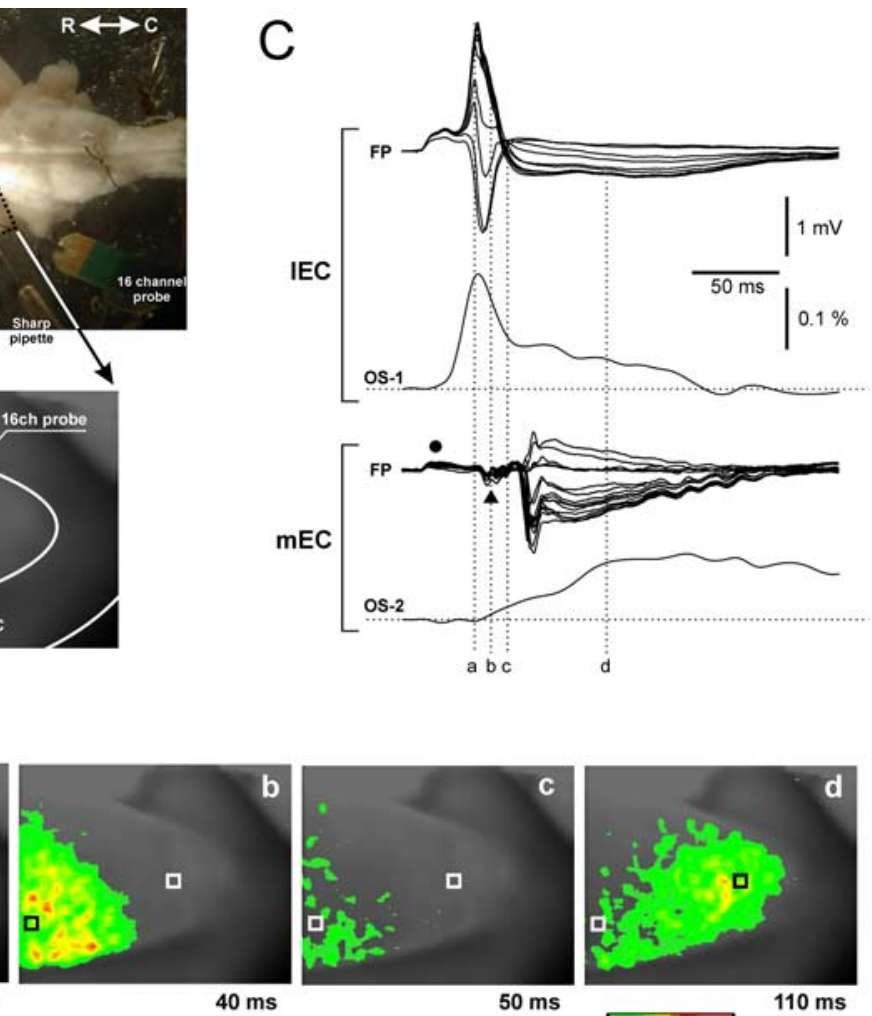

$40 \mathrm{~ms}$

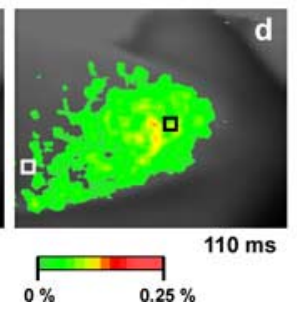

Figure 1. Identification of the EC area activated by the hippocampal output generated by $0.2-2 \mathrm{~Hz}$ stimulation of the lateral preparation via the arterial system with the voltage-sensitive dye di-2-ANEPEQ. Optical signals were recorded with a high-speed (he isolated brain with the position of the activation of the lateral and rostral portion of the EC $(\boldsymbol{B a}, \boldsymbol{B} \boldsymbol{b})$ is shown, followed by a delayed (>50 ms) activation of the (Biella et al., 2003; Gnatkovsky et al., 2004). The correlation between laminar profiles recorded with 16-channel silicon probe in the lateral EC (I-EC) and in the $\mathrm{m}$-EC and the optical signal recorded in I-EC (OS1) and in $\mathrm{m}$-EC (OS2) is

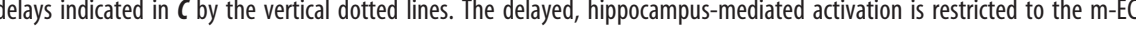
According to a previous study (de Curtis et al., 1999), the optical signal is most probably generated from neurons located in superficial layers. Optical recordings and laminar profiles in C were not recorded simultaneously.

ments, Milan, Italy) and were acquired and analyzed with ELPHO software developed by Vadym Gnatkovsky at the Istituto Nazionale Neurologico (Milan, Italy). Simultaneous intracellular recordings and extracellular recordings with silicon probes were performed in the medial EC. Current source density (CSD) analysis of laminar field profiles was performed with the 16-channel silicon probes on $200 \mu \mathrm{m}$ spacing according to the standard procedure (Mitzdorf, 1985; Ketchum and Haberly, 1993; de Curtis et al., 1994; Biella and de Curtis, 2000).

Optical recordings of the signal generated by cerebral cells stained with a voltage-sensitive dye were performed according to the technique described previously (Tominaga et al., 2000). The fast-response voltagesensitive dye di-2-ANEPEQ (1.5 mg) (Invitrogen, Eugene, OR) was arterially perfused for $2 \mathrm{~h}$. The dye is absorbed by neurons and the changes in fluorescence properties in response to change in membrane potential were recorded. A single staining of the brain allowed recording of the evoked fluorescent signal for 3-4 h. Optical signals were acquired with the MiCAM-01 charge-coupled device-based digital high-speed camera system (SciMedia, Irvine, CA; developed by BrainVision, Tsukuba, Japan).

At the end of the electrophysiological experiments, brains were removed from the incubation chamber and were fixed by immersion in a 

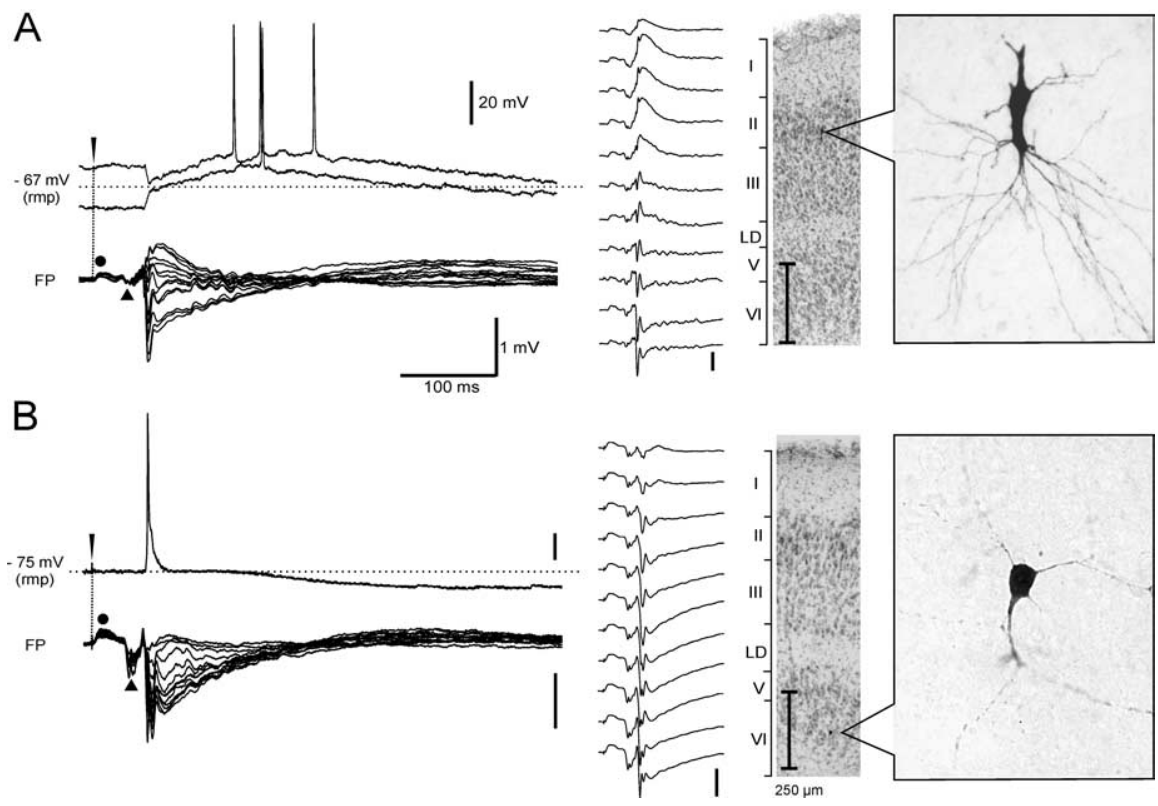

Figure 2. Typical electrophysiological responses recorded in a superficial $(\boldsymbol{A})$ and a deep $(\boldsymbol{B})$ layer $\mathrm{m}$-EC neuron during hippocampus-mediated activation induced by $0.2-2 \mathrm{~Hz}$ LOT stimulation. In the left part of each panel, the intracellular potentials (top traces) and the superimposed extracellular traces that form an FP laminar profile recorded with 16-channel silicon probes are illustrated. Intracellular recordings, performed at membrane potentials depolarized (top trace) and hyperpolarized (bottom trace) with respect to the resting membrane potential (rmp; dotted line), are shown for the superficial cell in $\boldsymbol{A}$. In the right part of each panel, the morphology of the recorded neurons, revealed by biocytin-HRP staining (right) and the position of the cell on thionine counterstained sections (left), are shown. Field responses within a laminar profile obtained at the indicated different depths are illustrated to the left of the histology sections. High-magnification pictures of the neurons were taken before performing the thionine staining.

$4 \%$ paraformaldehyde solution. After at least $24 \mathrm{~h}, 75-100 \mu \mathrm{m}$ slices were cut by Vibratome and the morphology of intracellularly recorded neurons was revealed by processing the sections with an $\mathrm{ABC}$ kit (Vector Laboratories, Burlingame, CA) for standard biocytin-horseradish peroxidase visualization. Sections were counterstained with thionine to identify cortical layers.

Bicuculline methiodide (1 mM) and ( \pm )-2-amino-5-phosphonopentanoic acid (AP-5; $2 \mathrm{~mm}$ ) were locally applied by pressure ejection via a blunted glass pipette; $100 \mu \mathrm{M}$ AP-5, 3 mM bicuculline, and a mixture of saclofen $(200 \mu \mathrm{M})$ and phaclofen $(500 \mu \mathrm{M})$ were also applied by arterial perfusion. Drugs were obtained from Sigma-Aldrich (St. Louis, MO).

\section{Results}

As reported previously (Biella and de Curtis, 2000; Biella et al., 2003; Gnatkovsky et al., 2004), repetitive stimulation of the LOT induces a delayed EC response generated by the hippocampal output (Uva and de Curtis, 2005). To localize the region of distribution of the hippocampal input in the EC, we performed a preliminary study by recording neuronal activity during patterned LOT stimulation with combined electrophysiological and imaging techniques (Fig. 1) $(n=4)$. The correlation with the optical signals generated during perfusion of the isolated brain with voltage-sensitive fast-response probe di-2-ANEPEQ demonstrated a direct response in the lateral-rostral part of the EC (l-EC) with a circa $30 \mathrm{~ms}$ delay (Fig. $1 \mathrm{Ba}, \mathrm{Bb}$ ). Such activation was followed by a response with a delay longer than $50 \mathrm{~ms}$ that invaded exclusively in the caudal and medial part of the EC (m-EC) (Fig. $1 B d$ ). According to a previous study (de Curtis et al., 1999), the optical signals recorded in the EC are most probably generated from neurons located in superficial layers. The correlation between the optical recordings (OS) and the field profiles (FP) with 16-channel silicon probes is illustrated in Figure 1C. Laminar depth reversals of field responses confirmed that the early activity was locally generated within the l-EC (Fig. $1 C$, right top panel), whereas the delayed response originated in the $\mathrm{m}-\mathrm{EC}$ (Fig. $1 C$, right bottom panel). The local $\mathrm{m}$-EC response was preceded by a complex far field that represents the sequential activation of the l-EC (filled dot) and the hippocampus (filled triangle) (Biella and de Curtis, 2000; Gnatkovsky et al., 2004; Uva and de Curtis, 2005). These data confirmed that, exclusively, the $\mathrm{m}$-EC receives the hippocampus-mediated output (Uva et al., 2004), a finding recently corroborated by an in vivo study (Kloosterman et al., 2003b). Therefore, we performed additional extracellular and intracellular electrophysiological recordings in this region.

Seventy-one and $20 \mathrm{~m}$-EC principal neurons were recorded with sharp electrodes in superficial (200-700 $\mu \mathrm{m}$ depth) and deep m-EC layers (800-1100 $\mu \mathrm{m}$ depth). Average membrane potentials of superficial and deep neurons were $-60.8 \pm 6.8 \mathrm{mV}$ and $-61.4 \pm 6.4 \mathrm{mV}$, respectively (mean $\pm \mathrm{SD}$ ). The morphology of cells was reconstructed after visualization of intracellularly injected biocytin. Pyramidal and stellate cells (Germroth et al., 1989; Lingenhöhl and Finch, 1991; Gloveli et al., 1997a; Klink and Alonso, 1997; van der Linden and Lopes da Silva, 1998) were recorded in superficial layers II and III. Polymorphic-multipolar and pyramidal neurons were found in deep layers V and VI (Hamam et al., 2000). The morphological classification of neuronal type was based on somatic and dendritic structures.

Both superficial and deep cells were activated exclusively in coincidence with the delayed hippocampus-mediated extracellular response, which was simultaneously recorded with a 16channel silicon probe in a position close to the intracellular recording electrode. The correlation between intracellular responses of typical superficial and deep neurons and FP laminar profiles recorded with the silicon probe is illustrated in Figures 2, 3 , and 4 .

\section{Deep neurons}

Deep layer neurons generated an excitatory potential that reached the firing threshold at resting membrane potential, as illustrated in Figures 2 B, 3, and 5. The onset of the intracellular response correlated with the onset of the deepest sink at $800-$ $1000 \mu \mathrm{m}$, detected by CSD analysis of the field potential laminar profile simultaneously performed with the silicon probe (Fig. 3, bottom). The depolarization that subtends deep cell action potentials increased in amplitude with membrane hyperpolarization (Fig. $5 A$ ) and was followed by a biphasic inhibitory potential that showed membrane potential reversals typical of $\mathrm{GABA}_{\mathrm{A}}$ $(-72.6 \pm 2.4 \mathrm{mV} ; n=17)$ and $\mathrm{GABA}_{\mathrm{B}}$-mediated IPSPs $(-98.7 \pm$ $3.9 \mathrm{mV} ; n=17$ ) (Fig. $5 B$ ). These findings suggest that deep neurons are briefly excited by the hippocampal output and are efficiently inhibited by a pronounced IPSP (see Discussion).

The onset of the EPSPs recorded in 20 deep m-EC neurons was plotted on the averaged time duration of the deep layer sinks calculated on the laminar profiles (Fig. 6, open circles). In $75 \%$ of 

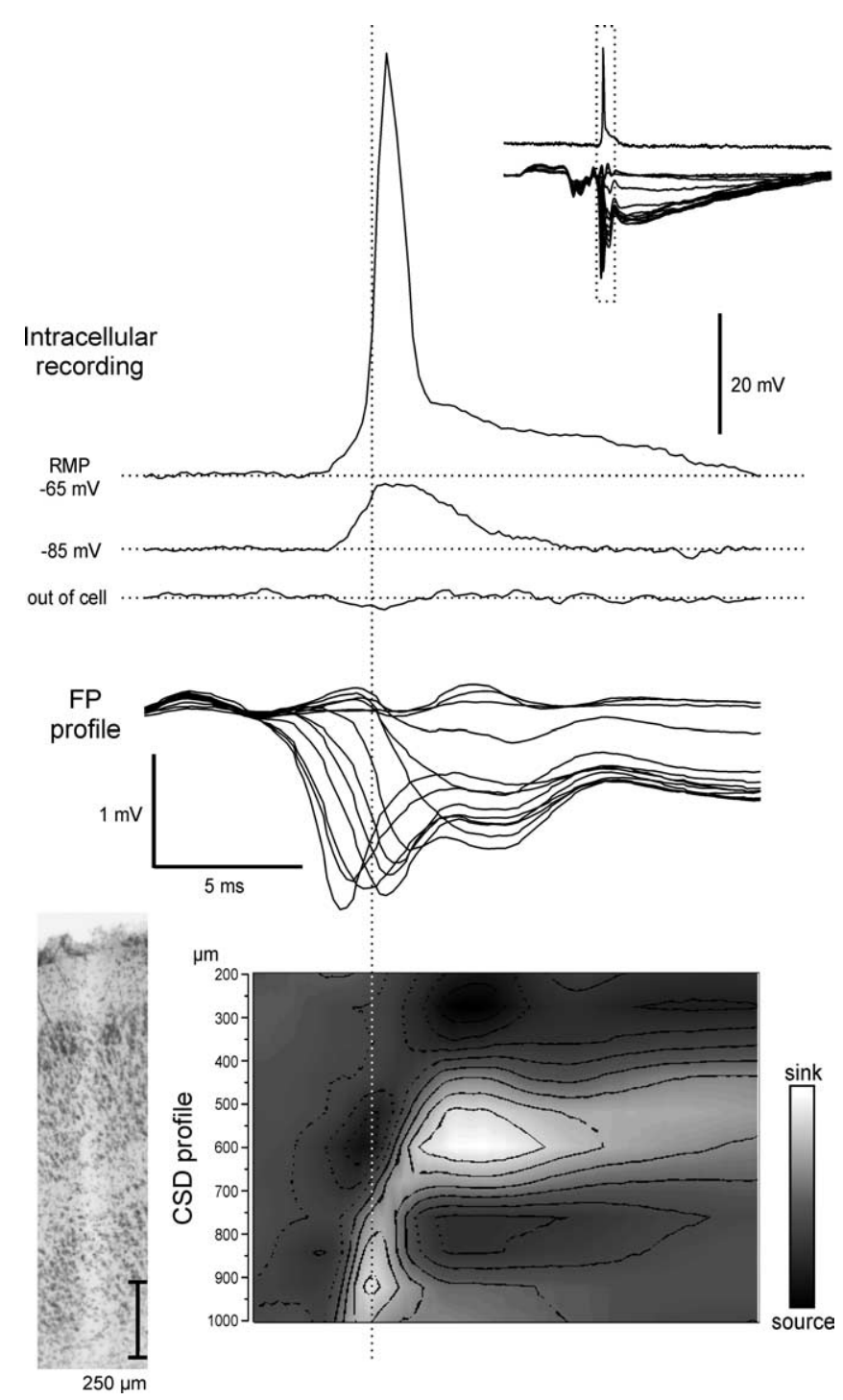

Figure 3. Correlation between intracellular activity of a deep m-EC neuron and the field potential laminar profile simultaneously recorded with a 16-channel silicon probe during $0.2-2$ $\mathrm{Hz}$ LOT stimulation. Intracellular recordings, performed at different membrane potentials after intracellular injection of steady current, are correlated to superimposed traces of the FP laminar profile and to the CSD profile (bottom; surface on top). A trace that shows the activity recorded with the intracellular electrode just before cell impalement is also shown ("out of cell") as control to exclude possible influences of the extracellular field potential on the intracellular responses; no field activity was recorded with the high-impedance intracellular electrode. In the top right inset, the intracellular and the extracellular traces are shown at a faster time scale. The track of the silicon probe is shown in the histological microphotograph to the left of the CSD profile. RMP, Resting membrane potential. Current calibration: from 0.3 to $-0.3 \mathrm{mV} / \mathrm{mm}^{2}$.

deep neurons, the onset of the EPSP coincided with the deepest $\mathrm{m}-\mathrm{EC}$ sink at $800-1000 \mu \mathrm{m}$.

\section{Superficial neurons}

In correlation to the simultaneously recorded delayed extracellular m-EC response, all superficial neurons generated an inhibitory potential followed by a prolonged depolarization (Fig. $2 \mathrm{~A}, 4$, and 7). None of the superficial neurons fired a spike before the inhibitory potential. The $\mathrm{GABA}_{\mathrm{A}}$-receptor antagonist bicuculline, applied by either arterial perfusion ( $3 \mathrm{~mm} ; n=5)$ or local injection in superficial layers ( $1 \mathrm{~mm} ; n=7)$, abolished the early inhibitory potential in m-EC superficial cells (Fig. 7A). Moreover, the early hyperpolarizing event reverted into a depolarizing potential when intracellular recordings were performed with micropipettes filled with $3 \mathrm{M} \mathrm{KCl}(n=5)$ (data not shown). Therefore, we identified such a potential as an IPSP that could be studied in isolation when the delayed depolarizing potential was abolished by both arterial $(100 \mu \mathrm{M})$ and local $(2 \mathrm{mM})$ applications of AP-5 (Fig. $7 \mathrm{~B}, \mathrm{C}$ ). In these conditions, the IPSP lasted $98.3 \pm$ $5.4 \mathrm{~ms}$ and showed a membrane reversal potential at $-72.4 \pm 2.3$ $\mathrm{mV}$ (Fig. $7 C)(n=6)$. A slow IPSP component with a more hyperpolarized membrane reversal $(-96.3 \pm 4.2 \mathrm{mV} ; n=6)$ was revealed in superficial cells by blocking the delayed depolarization by either arterial or local application of AP-5 (100 and $2 \mathrm{~mm}$, respectively) (Fig. $7 B-D$ ). The slow IPSP was blocked by arterial perfusion with a mixture of $\mathrm{GABA}_{\mathrm{B}}$ receptor antagonists phaclofen $(500 \mu \mathrm{M})$ and saclofen $(200 \mu \mathrm{M} ; n=3)$ (Fig. 7D).

Simultaneous intracellular recording and CSD analysis of laminar profiles demonstrated a correlation between the IPSP and a current sink located at 500-800 $\mu \mathrm{m}$ depth (Fig. 4). The onset of the inhibitory potential followed the deepest m-EC sink at $800-1000 \mu \mathrm{m}$ (Fig. $4 B$ ). The time relation between the IPSP onset recorded in 67 neurons and the averaged duration of the extracellular deep-layer sink is illustrated by the filled squares in Figure 6. The plot confirmed that the onset of the superficial cell IPSPs correlates to the sink at $500-800 \mu \mathrm{m}$.

The delayed depolarizing potential that follows the IPSP had a duration of $409 \pm 32.6 \mathrm{~ms}$ at resting membrane potential and was abolished by arterial perfusion of AP-5 (100 $\mu \mathrm{M} ; n=6)$ (Fig. $7 B, C)$, suggesting a dependence from synaptic NMDA receptor subtypes. The duration of such a slow EPSP decreased with membrane hyperpolarization (Figs. $4 A, 7 C$ ). It increased in amplitude and generated neuronal firing at $20-40 \mathrm{~Hz}$ when the membrane potential was depolarized from resting (Figs. 4A, 7C). The delayed slow EPSP was coupled to a superficial sink at $200-400 \mu \mathrm{m}$, identified by simultaneous recording of a laminar profile (Fig. 4A).

To exclude the possibility that the potentials recorded in superficial m-EC cells could be mediated by the propagation of activity within the $\mathrm{EC}$ via intrinsic associative connections that originate from the l-EC, we performed simultaneous extracellular recordings from $1-\mathrm{EC}, \mathrm{CA} 1$ in the hippocampus, and $\mathrm{m}-\mathrm{EC}$ after interrupting l-EC-to-m-EC associative fibers with a transverse blade cut, as illustrated in Figure $8 A(n=4)$. In these experimental conditions, the same pattern described above was observed in the extracellular field responses recorded in the CA1 region and in the lateral and medial EC before and after the cut (Fig. 8A). Simultaneous extracellular recording in $\mathrm{m}$-EC and intracellular recording in a superficial $\mathrm{m}$-EC neuron confirmed the persistence of the delayed responses after the disconnection between l-EC and m-EC (Fig. 8C). The intracellularly stained m-EC neuron is shown in the parasagittal section shown in Figure $8 D$ and in the expanded panel. The lesion between the l-EC and the m-EC (asterisk) is evident in Figure $8 B$ and $D$. The orientation of the cut is also illustrated by a dotted line in the schemes in Figure $8 A$ and $C$. The results confirmed that the delayed $\mathrm{m}$-EC activities are not generated by intra-EC associative connections.

\section{Discussion}

The isolated guinea pig brain is ideal for studying long-range and local neuronal interactions in unrestricted brain networks. We used this preparation to describe the EC network activated by the hippocampal output by means of optical imaging and intraextracellular electrophysiological recordings. To avoid retrograde EC activation, instead of directly stimulating the hippocampus output, we induced hippocampal responses by 
A

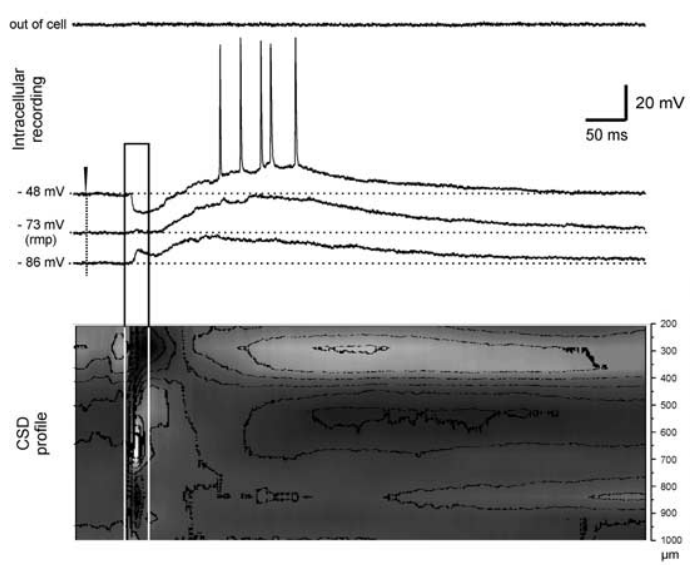

B

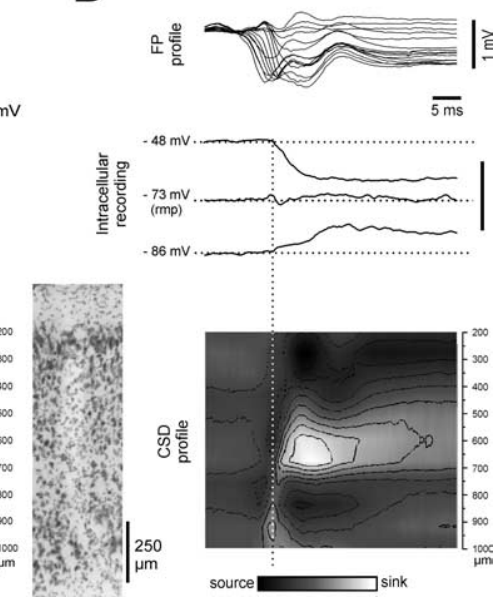

Figure 4. Correlation between intracellular activity of a superficial $m-E C$ neuron and the laminar profile simultaneously recorded with a 16-channel silicon probe during $0.2-2 \mathrm{~Hz}$ LOT stimulation. $\boldsymbol{A}$, Intracellular recording performed at different membrane potentials after intracellular injection of steady current. The activity recorded just out of the cell before intracellular penetration is illustrated on top ("out of cell"). The CSD profile is illustrated in the bottom part of the panel with the same time scale (surface on top). Superimposed traces of the FP laminar profile are illustrated in $\boldsymbol{B}$ (top) to enhance the correlation between intracellular signals, field potentials, and sink/sources. The time fragment outlined by the continuous line in $\boldsymbol{A}$ is $\operatorname{expanded}$ in $\boldsymbol{B}$. The track determined by the silicon probe is shown between $\boldsymbol{A}$ and $\boldsymbol{B}$, close to the CSD profile. rmp, Resting membrane potential. Current calibrations: $\boldsymbol{A}, \boldsymbol{B}$, from 0.3 to $-0.3 \mathrm{mV} / \mathrm{mm}^{2}$.

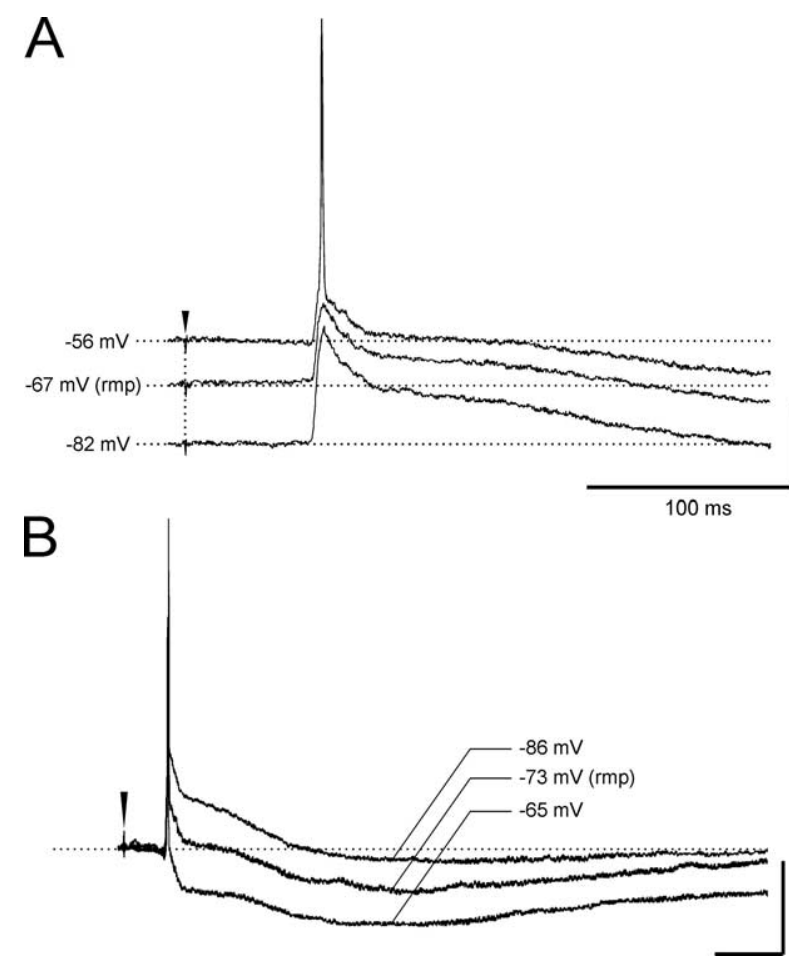

Figure 5. Dependence from membrane potential polarization and reversals of synaptic potentials in a typical deep $\mathrm{m}$-EC cell. The voltage dependence of the early EPSP is illustrated in $\boldsymbol{A}$. The reversals of the fast and slow IPSPs in a different deep layer neuron are shown in $\boldsymbol{B}$. rpm, Resting membrane potential.

repetitively stimulating the LOT at low frequencies. Such a stimulation is known to induce a delayed response in the $\mathrm{m}$-EC that is mediated by the hippocampal output (Biella et al., 2003; Gnatkovsky et al., 2004; Uva and de Curtis, 2005).

The present study demonstrates that the hippocampal output (1) generates EPSPs followed by a prolonged inhibition in deep

layer EC neurons, (2) transiently inhibits superficial EC neurons, possibly via a feedforward inhibitory circuit, and (3) generates a delayed synaptic excitation of superficial neurons that depends on NMDA-receptor activation and follows the IPSP. The network mechanisms that subtend such responses are discussed in the following paragraphs.

The circuit activated by LOT stimulation is schematically summarized in Figure 9 (Uva and de Curtis, 2005). LOT stimulation activates superficial neurons in the piriform cortex (PC), which are connected via associative fibers to the superficial layers (mostly layer II) of the rostral part of the l-EC (Krettek and Price, 1977; Wilson, 1978; Boeijinga and Van Groen, 1984; Liu and Bilkey, 1997; Biella and de Curtis, 2000; Biella et al., 2003). Stellate (and pyramidal) neurons of the l-EC project to the dentate gyrus (and the $\mathrm{CA}$ region) of the hippocampus, from where activity propagates to CA1 and to the subiculum (Witter, 1993). Pyramidal neurons in both these hippocampal subfields represent the main source of feedback output into the EC, which is largely relayed to the $\mathrm{m}$-EC in the guinea pig (Biella et al., 2003; Gnatkovsky et al., 2004) and in the rat (Kloosterman et al., $2003 \mathrm{~b}$ ). The observed $\mathrm{m}$-EC responses in superficial layer neurons could be caused by propagation of activity along associative fibers that originates from the 1-EC (Dolorfo and Amaral, 1998; Biella et al., 2002), directly activated by LOT stimulation. A possible role played by this projection in the network analyzed in the present study is ruled out by the experiments in which associative connections are severed by a transversal cut performed between 1-EC and m-EC.

Our study confirmed previous anatomical (HjorthSimonsen, 1971; Swanson and Cowan, 1977; Sorensen and Shipley, 1979; Tamamaki and Nojyo, 1995; Ino et al., 2001) and physiological findings (Kohler, 1985; Finch et al., 1986; Van Groen and Lopes da Silva, 1986; Jones, 1987; Bartesaghi et al., 1989; Kloosterman et al., 2003b, 2004), and demonstrates that the hippocampal input into the $\mathrm{m}$-EC excite neurons in the deep layers $\mathrm{V}$ and VI. The deep neuron EPSP/spike correlated with a current sink at 800-1000 $\mu \mathrm{m}$ depth (layers V-VI), which represents the first population event generated in the $\mathrm{m}$-EC after the hippocampal response (Bartesaghi et al., 1989; Kloosterman et al., 2003b). As shown during in vivo experiments (Finch et al., 1988), but not in EC slices (Jones, 1993), excitatory potentials in deep neurons were followed by biphasic IPSPs. Long-lasting feedback inhibition of deep EC cells after hippocampal output stimulation has been hypothesized previously by Kloosterman et al. (2003b).

Tracer studies demonstrated that a fraction of the efferent fibers that originate from CA1 and the subiculum also project to superficial m-EC layers (Dolorfo and Amaral, 1998; Ino et al., 2001; Kloosterman et al., 2003a; van Haeften et al., 2003). The existence of such a connection was suggested in vivo studies (Finch et al., 1986; Kloosterman et al., 2003b) and is definitively demonstrated in our electrophysiological experiments. The earliest response observed in superficial layer m-EC neurons was an inhibitory potential that (1) showed typical duration and membrane potential reversal of an IPSP mediated by GABAa recep- 


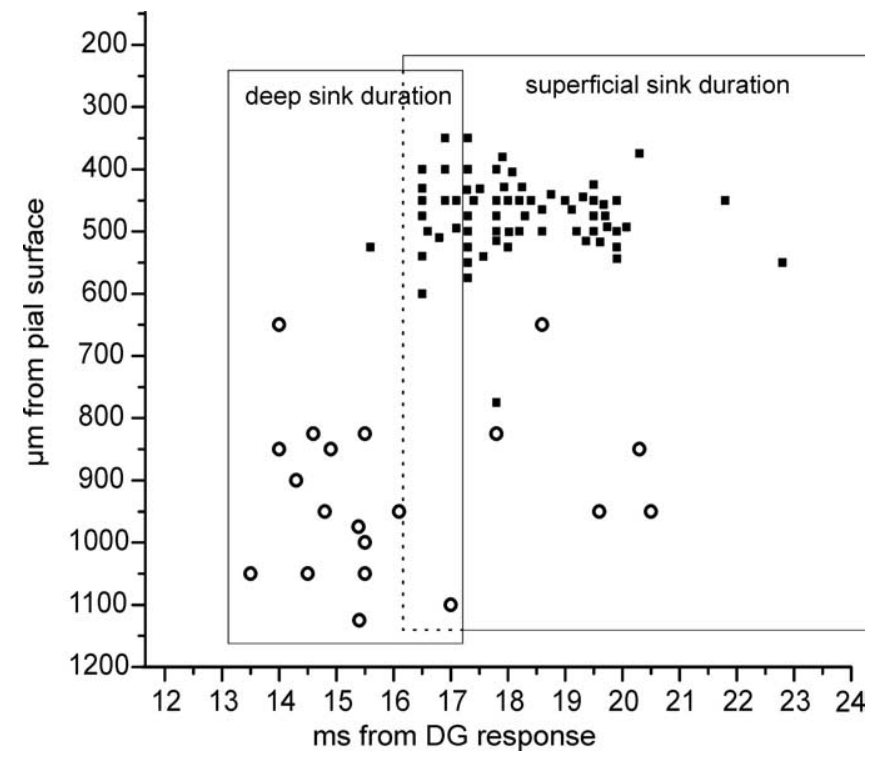

Figure 6. Correlation between the sinks recorded with 16-channel silicon probes and the onset of superficial cell IPSPs (filled squares) and deep cell EPSPs (open circles). The position of the symbols on the $y$ axis represents the depth of the neuron from which the synaptic potential was recorded. The time extensions of the deep and superficial sinks located at $800-1000 \mu \mathrm{m}$ and at $500-800 \mu \mathrm{m}$, respectively, are shown by the rectangular areas. The average duration of each sink was calculated on 14 experiments. Note the area of overlap between the two sinks.

tors, (2) appeared as a depolarizing event when recorded with $\mathrm{KCl}$-filled electrodes, and (3) was blocked by local application of bicuculline. The time course of the IPSP was characterized in detail when polysynaptic excitatory responses were abolished by NMDA-receptor antagonist treatment. Neither action potential firing nor excitatory synaptic potentials preceded the IPSP in superficial neurons, suggesting that it was generated by a feedforward inhibitory circuit. Feedforward IPSPs mediated by local interneurons were recorded during subicular and intraentorhinal stimulation on in vitro EC slices (Jones, 1993; Funahashi and Stewart, 1998) and have been demonstrated during local stimulation in vivo in the subicular area and in the EC (Finch et al., 1988). The activation of local GABAergic neurons in superficial m-EC (Jones and Buhl, 1993) via the hippocampal input fibers may be responsible for the generation of feedforward IPSPs. The most likely circuit responsible for feedforward inhibition in principal m-EC neurons of superficial layers is mediated by a direct activation of inhibitory interneurons (possibly located in superficial layers) mediated by the hippocampal output (Fig. 9). Intracellular and juxtacellular recordings from presumed EC interneurons performed in vivo (Finch et al., 1986) showed shortlatency bursting activity after hippocampal stimulation. Similar high-frequency firing was observed during preliminary intracellular recordings from fast-spiking neurons (putative interneurons) in the isolated guinea pig brain, in temporal coincidence with the earliest potential generated by the hippocampal output in m-EC deep layers (V. Gnatkovsky and M. de Curtis, unpublished observations).

As illustrated in Figure 6, the onset of superficial layer IPSPs showed a 2-3 ms delay from the EPSP generated in deep layer cells, suggesting that the IPSPs occurred with a one-synapse delay from deep cells activation. Because deep neurons are expected to exert mainly an excitatory effect on superficial neurons, the possibility that the IPSP in superficial neurons could possibly be mediated by the activation of deep EC cells is excluded. A small
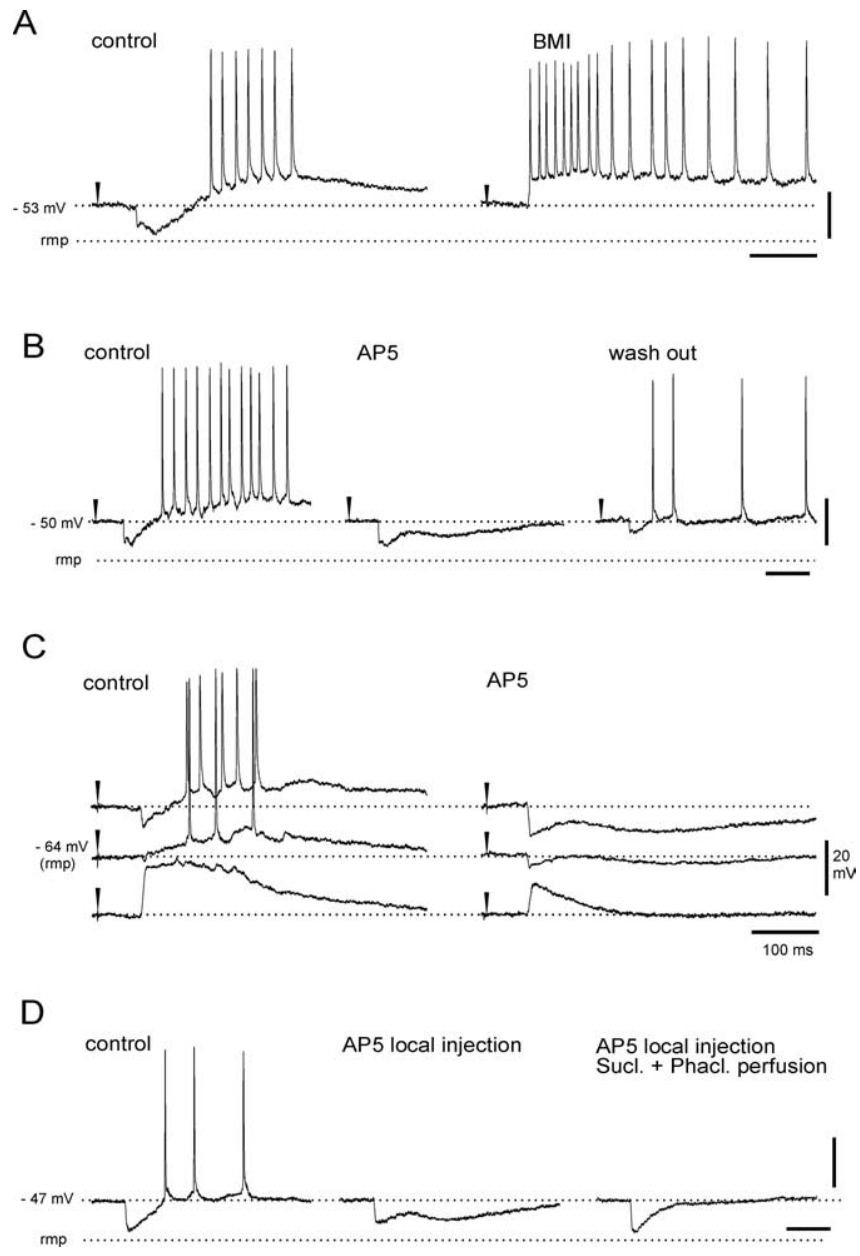

Figure 7. Characterization of the IPSP and the delayed slow EPSP in superficial $m-E C$ neurons. $A$, Local pressure ejection of the $G A B A_{A}$ receptor antagonist bicuculline methiodide (BMl; $1 \mathrm{~mm})$ via a blunted micropipette in the $\mathrm{m}-\mathrm{EC}(0.5 \mathrm{~mm}$ from the intracellular recording site) abolishes the early IPSP in a superficial neuron. $\boldsymbol{B}$, Arterial perfusion of the isolated brain with the NMDA receptor antagonist AP-5 $(100 \mu \mathrm{m})$ reversibly abolishes the delayed slow EPSP. C, Membrane potential dependence of both the IPSP and delayed slow EPSP. Perfusion with AP-5 reveals the time course and the reversal potential of the IPSP. D, Local pressure injection of AP-5 $(2 \mathrm{~mm})$ in the $\mathrm{m}$-EC abolishes delayed slow EPSP and reveals IPSPs. The late hyperpolarizing potential was abolished by combined arterial perfusion of $\mathrm{GABA}_{B}$ receptor antagonists saclofen $(200 \mu \mathrm{M})$ and phaclofen $(500 \mu \mathrm{M})$. rmp, Resting membrane potential.

proportion of excitatory synapses formed by deep EC axons also terminate on superficial interneurons (van Haeften et al., 2003). Superficial cell IPSPs mediated via deep neurons along such disynaptic circuits are expected have a longer delay than that observed in our experiments. This possibility is, therefore, also excluded.

The delayed slow depolarization observed in superficial neurons after the feedforward IPSP is likely to be synaptically mediated by intrinsic $\mathrm{m}$-EC connections activated by the hippocampal output. The effects of arterial and local intra-EC application of the antagonist of the NMDA-glutamate receptors, AP-5, suggest that this potential is mediated by an NMDA-dependent EPSP, although we cannot exclude that other conductances may contribute to its late part. Prominent NMDA-dependent EPSPs have been demonstrated in superficial EC neurons in vitro (Jones, 1994). Based on the analysis of time delays of synaptic responses, the most likely source of such an excitation originates from the activation of deep layer cells, which project to superficial cells via excitatory synapses (van Haeften et al., 2003). Alternatively, the 

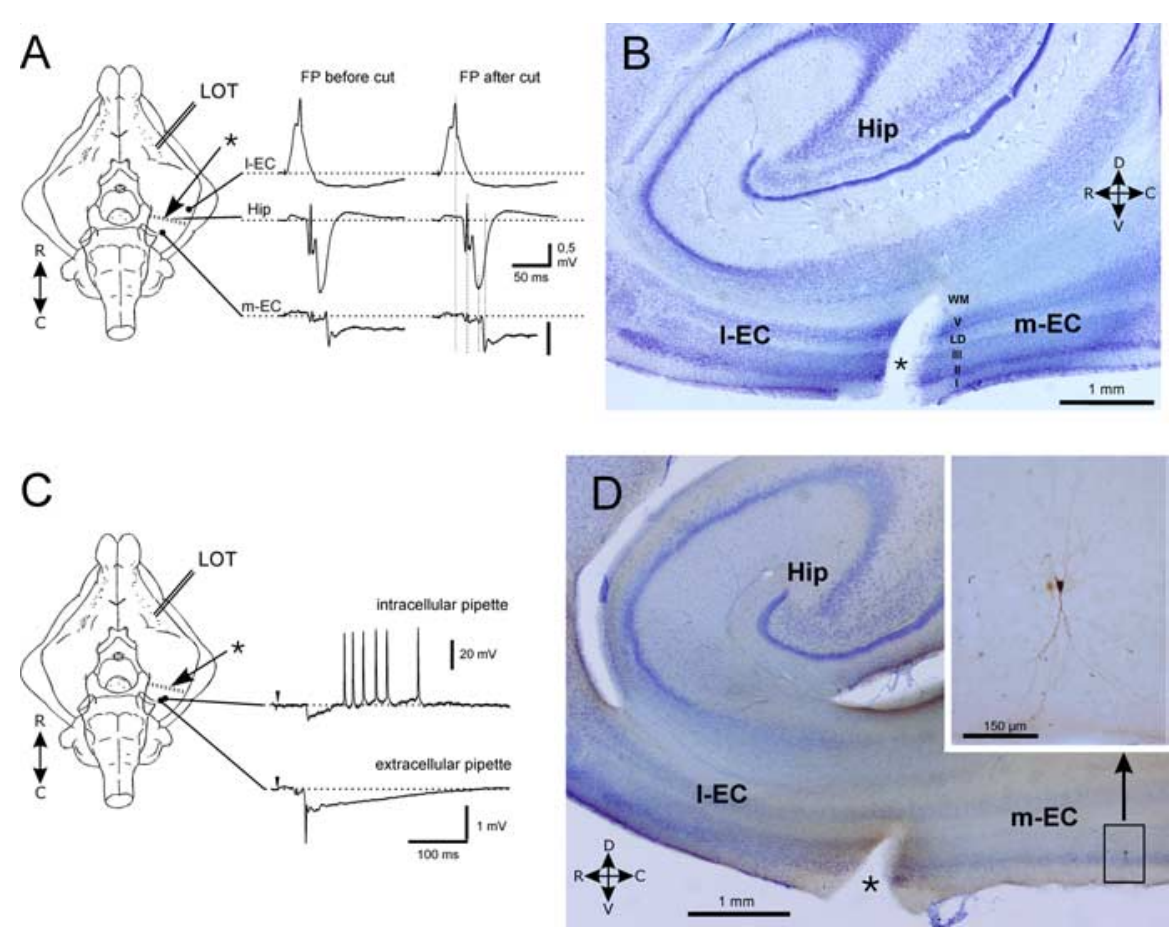

Figure 8. Delayed responses are not mediated by intra-EC associative fibers, which connect the I-EC to the m-EC. $A$, Scheme of the experimental setting. A blade cut was performed transversally in the EC to separate the I-EC from the $\mathrm{m}-\mathrm{EC}$ (dotted line marked by the asterisk). A thin foil of plastic was inserted into the cut to improve isolation. Simultaneous FP recordings in the I-EC, hippocampus (Hip; CA1), and $\mathrm{m}$-EC show no difference before and after cut. B, Parasagittal section (100 $\mu \mathrm{m})$ of the EC that includes the I-EC (left to cut) and the $\mathrm{m}$-EC (right to cut). The blade cut that interrupts the connections between the two $\mathrm{EC}$ subfields is marked by the asterisk. C, In a different experiment, simultaneous extracellular recordings and intracellular recording performed from a superficial $\mathrm{m}$-EC neuron after $\mathrm{m}$-EC disconnection show the same pattern observed in the intact preparation. $\boldsymbol{D}_{\text {, }}$ Parasagittal section $(100 \mu \mathrm{m})$ of the EC. The blade cut between the I-EC and $\mathrm{m}-\mathrm{EC}$ is marked by the asterisk. The superficial $\mathrm{m}$-EC pyramidal cell recorded in $C$ was revealed after visualizing the intracellularly injected biocytin. The position of the neuron in the $\mathrm{m}$ - $\mathrm{C}$ is illustrated in $\boldsymbol{B}$; its morphology is shown in detail in extended panel.

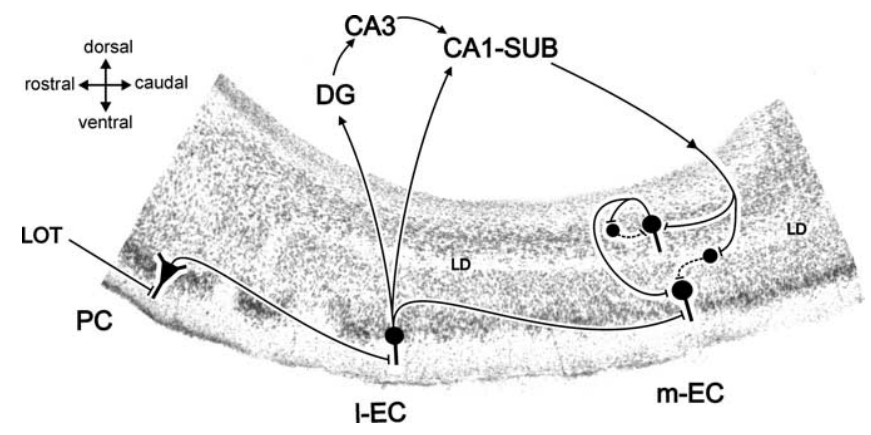

Figure 9. Summary of the hypothetical networks activated by the LOT-evoked hippocampal input into the $\mathrm{m}$-EC. The schematic representation of the circuit is superimposed on a parasagittal section of the olfactory-limbic cortex, which includes the PC, the I-EC, and the m-EC. See Discussion for details. Excitatory and inhibitory pathways are illustrated by continuous and dotted lines, respectively. LOT, Lateral olfactory tract; $\mathrm{PC}$, piriform cortex; $\mathrm{DG}$, dentate gyrus; $\mathrm{CA}$, cornu ammonis; LD, lamina dissecans; SUB, subiculum.

EPSP could be mediated by a direct excitatory effect of the hippocampal output from the CA1 and subiculum, activated in parallel to the direct excitation of interneurons responsible for the feedforward IPSP. If this were the case, the direct hippocampal excitation on superficial cells should precede, by one synaptic delay, the feedforward IPSP. The absence of an EPSP in superficial neurons before the feedforward IPSP plays against this possibility.

The delayed EPSP promotes firing that is not synchronous between neurons, because it does not generate clear synchronous population spikes. Preliminary findings suggest that firing generated during the slow EPSP may sustain oscillatory sequences similar to the periodic oscillations observed during muscarinic activation (Dickson et al., 2003) that promote cortical arousal and plasticity. It could be speculated that this mechanism of hippocampus-mediated non synchronous activation of superficial EC layers may play a role in setting a condition of EC arousal that facilitates integration with other converging inputs (i.e., the neocortically derived input mediated via the perirhinal/postrhinal cortex) (Burwell and Amaral, 1998; de Curtis and Pare, 2004). It is interesting to note that in our experimental condition, the m-EC activation is driven by a patterned lowfrequency stimulation that mimics the sniffing frequency used by lower mammals during olfactory discrimination (Macrides et al., 1982). As discussed previously, the selective $\mathrm{m}$-EC activation observed during such a peculiar stimulation pattern may support odor discrimination as well as olfactory-driven memory processes.

Both feedforward inhibition of superficial EC cells and desynchronization of firing during the delayed NMDA-dependent EPSP prevent massive activation of the EC-hippocampal reentrant pathway. $\mathrm{Re}$ verberation along the hippocampal-EChippocampal loop has never been shown in physiological conditions and is frequently associated to the generation of epileptiform discharges. Loop reverberation has been, indeed, observed exclusively during prolonged repetitive stimulation (Bartesaghi and Gessi, 2003; Kloosterman et al., 2004) and during epileptogenic conditions (Pare et al., 1992). Therefore, massive hippocampal-EC reverberation is possibly a nonphysiological event that should be prevented to avoid epileptiform hyperexcitability. The circuit described in the present study may be responsible for controlling pathologic synchronous reverberation in the EC-hippocampal loop and could play a role in regulating memory EC functions by favoring asynchronous superficial-layer firing rather than massive loop reverberation.

\section{References}

Bartesaghi R, Gessi T (2003) Activation of perforant path neurons to field CA1 by hippocampal neurons. Hippocampus 13:235-249.

Bartesaghi R, Gessi T, Sperti L (1989) Electrophysiological analysis of the hippocampal projections to the entorhinal area. Neuroscience 30:51-62.

Berretta N, Jones RSG (1996) A comparison of spontaneous EPSCs in layer II and layer IV-V neurons of the rat entorhinal cortex in vitro. J Neurophysiol 76:1089-1100.

Biella G, de Curtis M (2000) Olfactory inputs activate the medial entorhinal cortex via the hippocampus. J Neurophysiol 83:1924-1931.

Biella G, Uva L, Hoffman U, de Curtis M (2002) Associative potentials in the entorhinal cortex of the guinea pig. J Neurophysiol 88:1159-1165.

Biella GR, Gnatkovsky V, Takashima I, Kajiwara R, Iijima T, de Curtis M (2003) Olfactory input to the parahippocampal region of the isolated guinea pig brain reveals weak entorhinal-to-perirhinal interactions. Eur J Neurosci 18:95-101.

Boeijinga PH, Van Groen Th (1984) Inputs from the olfactory bulb and 
olfactory cortex to the entorhinal cortex in the cat. Exp Brain Res 57:40-48.

Burwell R (2000) The parahippocampal region: corticocortical interconnectivity (Scharfman HE, Witter MP, Schwarcz R, eds), pp 25-42. New York: New York Academy of Sciences.

Burwell RD (2001) The perirhinal and postrhinal cortices of the rat: borders and cytoarchitecture. J Comp Neurol 437:17-41.

Burwell RD, Amaral DG (1998) Perirhinal and postrhinal cortices of the rat: interconnectivity and connections with the entorhinal cortex. J Comp Neurol 391:293-321.

de Curtis M, Pare D (2004) The rhinal cortices: a wall of inhibition between the neocortex and the hippocampus. Prog Neurobiol 74:101-110.

de Curtis M, Pare D, Llinas RR (1991) The electrophysiology of the olfactory-hippocampal circuit in the isolated and perfused adult mammalian brain in vitro. Hippocampus 1:341-354.

de Curtis M, Biella G, Forti M, Panzica F (1994) Multifocal spontaneous epileptic activity induced by restricted bicuculline ejection in the piriform cortex of the isolated guinea pig brain. J Neurophysiol 71:2463-2475.

de Curtis M, Biella G, Buccellati C, Folco G (1998) Simultaneous investigation of the neuronal and vascular compartments in the guinea pig brain isolated in vitro. Brain Res Brain Res Protoc 3:221-228.

de Curtis M, Takashima I, Iijima T (1999) Optical recordings of cortical activity after in vitro perfusion of cerebral arteries with a voltage-sensitive dye. Brain Res 837:314-319.

Deadwyler SA, West JR, Cotman CW, Lynch G (1975) Physiological studies of the reciprocal connections between the hippocampus and entorhinal cortex. Exp Neurol 49:35-57.

Dickson CT, Mena AR, Alonso A (1997) Electroresponsiveness of medial entorhinal cortex layer III neurons in vitro. Neuroscience 81:937-950.

Dickson CT, Biella G, de Curtis M (2003) Slow periodic events and their transition to gamma oscillations in the entorhinal cortex of the isolated Guinea pig brain. J Neurophysiol 90:39-46.

Dolorfo CL, Amaral DG (1998) Entorhinal cortex of the rat: organization of intrinsic connections. J Comp Neurol 398:49-82.

Finch DM, Wong EE, Derian EL, Chen XH, Nowlin-Finch NL, Brothers LA (1986) Neurophysiology of limbic system pathways in the rat: projections from the amygdala to the entorhinal cortex. Brain Res 370:273-284.

Finch DM, Tan AM, Isokawa-Akesson M (1988) Feedforward inhibition of the rat entorhinal cortex and subicular complex. J Neurosci 8:2213-2226.

Funahashi M, Stewart M (1998) GABA receptor-mediated post-synaptic potentials in the retrohippocampal cortices: regional, laminar and cellular comparisons. Brain Res 787:19-33.

Germroth P, Schwerdtfeger WK, Buhl EH (1989) Morphology of identified entorhinal neurons projecting to the hippocampus. A light microscopical study combining retrograde tracing and intracellular injection. Neuroscience 30:683-691.

Gloveli T, Schmitz D, Empson RM, Dugladze T, Heinemann U (1997a) Morphological and electrophysiological characterization of layer III cells of the medial entorhinal cortex of the rat. Neuroscience 77:629-648.

Gloveli T, Schmitz D, Empson RM, Heinemann U (1997b) Frequencydependent information flow from the entorhinal cortex to the hippocampus. J Neurophysiol 78:3444-3449.

Gloveli T, Dugladze T, Schmitz D, Heinemann U (2001) Properies of entorhinal cortex deep layer neurons projecting to the rat dentate gyrus. Eur J Neurosci 13:413-420.

Gnatkovsky V, de Curtis M (2004) Olfactory tract-mediated hippocampal input to the entorhinal cortex of the isolated guinea pig brain: simultaneous profile and intracellular analysis. Soc Neurosci Abstr 30:775.1.

Gnatkovsky V, Uva L, de Curtis M (2004) Topographic distribution of direct and hippocampus-mediated entorhinal cortex activity evoked by olfactory tract stimulation. Eur J Neurosci 20:1897-1905.

Hamam BN, Kennedy TE, Alonso A, Amaral DG (2000) Morphological and electrophysiological characteristics of layer $\mathrm{V}$ neurons of the rat medial entorhinal cortex. J Comp Neurol 418:457-472.

Hjorth-Simonsen (1971) A Hippocampal efferents to the ipsilateral entorhinal area: an experimental study in the rat. J Comp Neur 142:417-438.

Ino T, Kaneko T, Mizuno N (2001) Projections from the hippocampal and parahippocampal regions to the entorhinal cortex. An anterograde and retrograde tract-tracing study in the cat. Neurosci Res 39:51-69.

Insausti R, Herrero MT, Witter MP (1997) Entorhinal cortex of the rat: cytoarchitectonic subdivisions and the origin and distribution of cortical efferents. Hippocampus 7:146-183.
Isokawa M, Finch DM (1991) Commissural responses of rat retrohippocampal neurons. Brain Res 551:94-103.

Jones RSG (1987) Complex synaptic responses of entorhinal cortical cells in the rat to subicular stimulation in vitro: demonstration of an NMDA receptor-mediated component. Neurosci Lett 81:209-214.

Jones RSG (1993) Entorhinal-hippocampal connections: a speculative view of their function. Trends Neurosci 16:58-64.

Jones RSG (1994) Synaptic and intrinsic properties of neurons of origin of the perforant path in layer II of the rat entorhinal cortex in vitro. Hippocampus 4:335-353.

Jones RSG, Buhl EH (1993) Basket-like interneurons in layer II of the entorhinal cortex exhibit a powerful NMDA-mediated synaptic excitation. Neurosci Lett 149:35-39.

Jones RSG, Heinemann V (1988) Synaptic and intrinsic responses of medial entorhinal cortical cells in normal and magnesium-free medium in vitro. J Neurophysiol 59:1476-1496.

Ketchum KL, Haberly LB (1993) Membrane currents evoked by afferent fiber stimulation in rat piriform cortex. I. Current source-density analysis. J Neurophysiol 69:248-260.

Klink R, Alonso A (1993) Ionic mechanisms for the subthreshold oscillations and differential electroresponsiveness of medial entorhinal cortex layer II neurons. J Neurophysiol 70:144-157.

Klink R, Alonso A (1997) Morphological characteristics of layer II projection neurons in the rat medial entorhinal cortex. Hippocampus 7:571-583.

Kloosterman F, Van Haeften T, Lopes Silva FH (2000) Functional characterization of hippocampal output to the entorhinal cortex in the rat. Ann NY Acad Sci 911:459-461.

Kloosterman F, Witter MP, Van Haeften T (2003a) Topographical and laminar organization of subicular projections to the parahippocampal region of the rat. J Comp Neurol 455:156-171.

Kloosterman F, Van Haeften T, Witter MP, Lopes Da Silva FH (2003b) Electrophysiological characterization of interlaminar entorhinal connections: an essential link for re-entrance in the hippocampal-entorhinal system. Eur J Neurosci 18:3037-3052.

Kloosterman F, van Haeften T, Lopes da Silva FH (2004) Two reentrant pathways in the hippocampal-entorhinal system. Hippocampus 14:1026-1039.

Kohler C (1985) Intrinsic projections of the retrohippocampal region in the rat brain. I. The subicular complex. J Comp Neurol 236:504-522.

Kohler C (1986) Intrinsic connections of the retrohippocampal region in the rat brain. II. The medial entorhinal area. J Comp Neurol 246:149-169.

Krettek JE, Price JL (1977) Projections from the amygdaloid complex and adjacent olfactory structures to the entorhinal cortex and the subiculum in the rat and cat. J Comp Neurol 172:723-752.

Lingenhöhl K, Finch DM (1991) Morphological characterization of rat entorhinal neurons in vivo: soma-dendritic structure and axonal domains. Exp Brain Res 84:57-74.

Liu P, Bilkey DK (1997) Parallel involvement of perirhinal and lateral entorhinal cortex in the polysynaptic activation of hippocampus by olfactory inputs. Hippocampus 7:296-306.

Llinás R, Yarom Y, Sugimori M (1981) Isolated mammalian brain in vitro: new technique for analysis of electrical activity of neuronal circuit function. Fed Proc 40:2240-2245.

Lopes da Silva FH, Witter MP, Boeijinga PH, Lohman AH (1990) Anatomic organization and physiology of the limbic cortex. Physiol Rev 70:453-511.

Macrides F, Eichenbaum HB, Forbes BW (1982) Temporal relationship between sniffing and the limbic theta rhythm during odor discrimination reversal learning. J Neurosci 2:1705-1717.

Mitzdorf U (1985) Current source-density method and application in cat cerebral cortex: investigation of evoked potentials and EEG phenomena. Physiol Rev 65:37-100.

Muhlethaler M, de Curtis M, Walton K, Llinas R (1993) The isolated and perfused brain of the guinea-pig in vitro. Eur J Neurosci 5:915-926.

Pare D, deCurtis M, Llinas R (1992) Role of the hippocampal-entorhinal loop in temporal lobe epilepsy: extra- and intracellular study in the isolated guinea pig brain in vitro. J Neurosci 12:1867-1881.

Sorensen KE, Shipley MT (1979) Projections from the subiculum to the deep layers of the ipsilateral presubicular and entorhinal cortices in the guinea pig. J Comp Neurol 188:313-334.

Squire LR, Zola SM (1996) Structure and function of declarative and nondeclarative memory systems. Proc Natl Acad Sci USA 93:13515-13522. 
Swanson LW, Cowan WM (1977) An autoradiographic study of the organization of the efferent connections of the hippocampal formation in the rat. J Comp Neurol 172:49-84.

Tamamaki N, Nojyo W (1995) Preservation of topography in the connections between the subiculum, field CA1 and the entorhinal cortex in rats. J Comp Neurol 353:379-390.

Tominaga T, Tominaga Y, Yamada H, Matsumoto G, Ichikawa M (2000) Quantification of optical signals with electrophysiological signals in neural activities of Di-4-ANEPPS stained rat hippocampal slices. J Neurosci Methods 102:11-23.

Uva L, de Curtis M (2005) Polysynaptic olfactory pathway to the ipsi- and contralateral entorhinal cortex mediated via the hippocampus. Neuroscience 130:249-258.

Uva L, Gruschke S, Biella G, De Curtis M, Witter MP (2004) Cytoarchitectonic characterization of the parahippocampal region of the guinea pig. J Comp Neurol 474:289-303.

van der Linden S, Lopes da Silva FH (1998) Comparison of the electrophysiology and morphology of layers III and II neurons of the rat medial entorhinal cortex in vitro. Eur J Neurosci 10:1479-1489.

Van Groen T, Lopes da Silva FH (1986) Organization of the reciprocal connections between the subiculum and the entorhinal cortex in the cat. II. An electrophysiological study. J Comp Neurol 251:111-120.

van Groen T, van Haren FJ, Witter MP, Groenewegen HJ (1986) Organization of the reciprocal connections between the subiculum and the ento- rhinal cortex in the cat: I. A neuroanatomical tracing study. J Comp Neurol 250:485-497.

van Haeften T, Jorritsma-Byham B, Witter MP (1995) Quantitative morphological analysis of subicular terminals in the rat entorhinal cortex. Hippocampus 5:452-459.

van Haeften T, Baks-te-Bulte L, Goede PH, Wouterlood FG, Witter MP (2003) Morphological and numerical analysis of synaptic interactions between neurons in deep and superficial layers of the entorhinal cortex of the rat. Hippocampus 13:943-952.

Wang X, Lambert NA (2003) Membrane properties of identified lateral and medial perforant pathway projection neurons. Neuroscience 117:485-492.

Wilson RCSO (1978) Polysynaptic activation of the dentate gyrus of the hippocampal formation: an olfactory input via the lateral entorhinal cortex. Exp Brain Res 33:523-534.

Witter MP (1993) Organization of the entorhinal-hippocampal system: a review of current anatomical data. Hippocampus 3 [Spec No]:33-44.

Witter MP, Wouterlood F (2002) The parahippocampal region. Organization and role in cognitive functions. Oxford: Oxford UP.

Witter MP, Groenewegen HJ, Lopes da Silva FH, Lohman AH (1989) Functional organization of the extrinsic and intrinsic circuitry of the parahippocampal region. Progr Neurobiol 33:161-253.

Yang S, Lee DS, Chung CH, Cheong MY, Lee CJ, Jung MW (2004) Longterm synaptic plasticity in deep layer-originated associational projections to superficial layers of rat entorhinal cortex. Neuroscience 127:805-812. 